\title{
A Diabetes Psychosocial Profile
}

GEORGE E. HESS, MA

WAYNE K. DAVIS, PhD

R. VAN HARRISON, PhD, all of the

Department of Postgraduate Medicine

and Health Professions Education

The University of Michigan Medical School

Ann Arbor, Michigan

An educational needs assessment instrument for individuals with diabetes has been developed at the Michigan Diabetes

Research and Training Center. Responses to the 110-item questionnaire provide subscores on constructs labeled "Control Problems,"' "Psychosocial Impact," "Barriers to Compliance," "Benefits of Regimen," "Regimen Complexity," and "Risk of Complications." Combined with demographic and clinical information, these scores form a comprehensive summary of the patient's attitudes, beliefs, behaviors, and knowledge in relation to diabetes. This summary is in the form of an individualized graphic profile that highlights possible problem areas to be considered when developing patient education and management plans.
The effectiveness of diabetes management to a very large extent is controlled by the patient rather than by the health care professional. Diabetes mellitus, especially when managed with insulin, cannot be treated successfully without the cooperation of well-informed, trained, and motivated patients. Clearly, a major goal of patient education must be to enhance the ability of patients to comply with their therapeutic regimens and to cope with the implications of a chronic disease and its impact on their lives. The demands placed on the patient are considerable; Surwit et al ${ }^{1,2}$ described in detail the behavioral complexities of diabetes self-management and the lack of clear rewards, which frequently presage compliance problems.

Dunbar and Stunkard, ${ }^{3}$ in a study of compliance in patients with hypertension, stated that the most important determinant of patient nonadherence to a therapeutic regimen is the complexity of the regimen itself. They emphasized the value of expert instruction in the conduct of the regimen, carefully individualized for the unique circumstances of each patient. Dudley ${ }^{4}$ agreed with this need for individualization of patient education and identified a thorough educational assessment as the foundation of effective patient education.

Health care professionals, a primary source of patient education, must be able to assess instructional needs as well as their patients' ability and willingness to assume responsibility for managing their therapeutic regimens. The goal of the project described in this article was to provide diabetes educators with an individualized needs assessment instrument for patients that was easily administered and interpreted, reliable, and valid. The parameters of this assessment included the demands placed on patients by their therapeutic regimen, their behavioral response to these demands, and the psychosocial components of life with a chronic disease. The Diabetes Educational Profile (DEP) was designed to measure and summarize an individual's attitudes, beliefs, and behaviors concerning diabetes and the related treatment regimen. A major objective during development of the profile was to present the resulting data to health care professionals in an easily understood manner that would be immediately applicable to patient management.

Acknowledgments The research reported within this article was supported, in part, by The National Institute of Arthritis, Diabetes, Digestive and Kidney Diseases, Michigan Diabetes Research and Training Center (NIH 2P60AM20572) and the Michigan Department of Public Health, Division of Chronic Disease Control.

Reprint requests to George E. Hess, MA, Program Manager, Department of Postgraduate Medicine and Health Professions Education, Office of Educational Resources and Research, G1113 Towsley Center, Box 057, The University of Michigan Medical School, Ann Arbor, MI 48109. 
To maximize validity, reliability, and utility, the DEP was made diabetes-specific, rather than nondisease-specific or generalizable to other chronic diseases. The rationale for this decision has been well expressed by Skyler ${ }^{5}$ and again by Glasgow and McCaul. ${ }^{6}$ Each of these authors questioned the utility of psychological assessment instruments standardized on the general population. All favored using measures constructed specifically to assess parameters relevant to individuals with diabetes.

In large part, the theoretical basis for the DEP is that of the Health Belief Model. ${ }^{7}$ Dunbar and Stunkard ${ }^{3}$ reviewed the application of this model, which relates beliefs to health actions, in a number of studies. For each belief category (Perceived Susceptibility, Perceived Severity, Perceived Benefits, Perceived Cost) they found support for the model when applied to specific groups or types of patients. The model was found to be more applicable to symptomatic than asymptomatic patients and to those having had some experience with the treatment in question. Although the rationale of the DEP parallels that of the Health Belief Model, the content and structure are wholly within the context of diabetes. It contains two major sections: (1) a demographic and psychosocial assessment, and (2) a diabetes health history.

The purposes of this article are to document the development of the instrument and its psychometric properties, and to describe the profile format that has been developed to present the data for clinical application. Data obtained from over 600 administrations of the Profile were used to accomplish the following: determine the empirical factor structure of the instrument, confirm the clinical relevance of each item, develop a scoring algorithm for each subscale, investigate the reliability and validity of the scales thus obtained, and design an easily interpreted, individualized patient summary that highlights possible problem areas to consider in developing patient education and management plans.

\section{Methods}

Instrument Development The first phase in the development of the DEP instrument involved identifying patient characteristics that, when known to the health provider, could lead to more effective educational and behavioral interventions. Knowledge of these characteristics and consequent adjustment in the care program were hypothesized to facilitate patient adherence. As previously reported, ${ }^{8}$ a literature review of some 250 journal articles resulted in the identification of more than 500 characteristics that were subsequently edited and refined to form a list of 144 different phrases describing patient characteristics stated in behavioral terms. A questionnaire based on this list was sent to over 1500 clinicians and health educators, who were asked to evaluate on a five-point scale the importance of each specific patient characteristic. The structure and content of the DEP were based on statistical analysis and review of 802 responses to this questionnaire by a team composed of health professionals, health educators, and behavioral scientists.

The first section of the DEP included eight demographic items and 50 psychosocial items. The second section (diabetes health history) contained 51 items: 12 concerning medical history, 28 on current diabetes management behaviors, and 11 forming a self-report of adherence. The constructs measured by these items closely paralleled similar constructs in the

\section{Table 1. Planned Structure of DEP Content}

Section 1: Demographic-Psychosocial Assessment Topic

Demographic barriers:

Diet

Urine test

Exercise

Benefits of Regimen

Adjustment

Social effects

Health attitudes

Social support

Risk of complications

Section 2: Diabetes Health History

Topic

Medical history

Management behaviors

Self-report of compliance

No. Items

8

7

3

2

6

8

7

10

4

No. Items

Health Belief Model, although each had been made specific to diabetes. Table 1 displays the content of these sections in greater detail.

Sample The DEP was pilot-tested in three Michigan communities. Questionnaries were completed by 201 patients under the care of diabetologists both in hospital and in outpatient settings. Data from these administrations were used to investigate the psychometric properties of the instrument and to develop a scoring system suitable for field use of the DEP.

During the course of a concurrent Michigan Diabetes Research and Training Center project, 428 additional administrations of the DEP were obtained from a randomly selected patient sample in eight Michigan communities. ${ }^{9}$ Physiological data and self-reports of health practices and outcomes also were available for this group of patients. The analyses reported here, unless otherwise noted, were based on this later sample-which is believed to be generally representative of the diabetic population in the state of Michigan.

Factor Structure Factor analysis was used to reveal the empirical structure of the instrument and to measure item quality. This structure, determined by grouping items according to statistically measured similarities in response pattern, provided subsets of items that measure similar content and yield the most reliable scores. An initial principal axis factor analysis was used to identify and eliminate from further analysis items not clearly associated with any of the resulting factors. The relationship of the included items to one another was clarified through a statistical transformation (rotation) of the previously determined principal axis structure. All composite scores used in further analysis of the DEP reflected the structure determined by this factor analysis.

Scoring and Psychometric Properties Simplicity of scoring was considered essential if the DEP were to be scored by hand rather than by computer. The ability to hand-score the DEP would allow the instrument to be made widely available for clinical use. Scoring was facilitated by assigning each item to the single factor on which it loaded most strongly. All of the 
items within a factor were weighted equally for scoring; thus, each item contributed equally to the total factor score. The direction in which each item was to be scaled was confirmed by calculating item-total correlations within each factor. Cronbach's coefficient alpha ${ }^{10}$ was used as a minimum estimate of the reliability of the factor scores thus obtained.

Content validity, which refers to the adequacy of the content of the instrument, was established by the process used to develop the DEP. Evidence for construct validity was developed by comparing the empirical structure determined by factor analysis of response patterns to the planned structure of the instrument based on the initial content outline. The criterion validity of the instrument was assessed by examining the relationship between profile scores and measures of health status and behavior.

Presentation of Individual Profiles The final step in developing the educational profile was to develop a useful and easily interpreted method of presenting these data to health professionals. The goal was to emphasize the individuality of each patient and to present data relevant to individualized instruction and counseling in a single, easily understood document. This meant that for each patient, demographic information, data on the prescribed regimen, health status, and educational history would need to be combined in a simple manner with the psychosocial assessment. It was also necessary to present the scale scores in a manner that would facilitate a clinician's understanding of their true significance. A number of presentation formats were developed. Feedback on their clarity and utility was obtained from health educators and physicians. Actual patient profiles were interpreted by diabetes clinicians and their comments formed the basis for revisions.

\section{Results}

Factor Structure The initial structural analysis of the DEP was performed using the pilot sample. Items from the instrument with at least ordinal properties ( 94 items) were included in a preliminary factor analysis. The results of this analysis were examined to identify and reject items for which response patterns were not significantly related (for example, loading less than .3 on any factor extracted) to those of other items. Twenty items were thus eliminated from further analysis.

The remaining 74 items were included in a series of statistical rotations of the factor matrix using the (promax [4]) algorithm to determine the solution most representative of the simple structure of the DEP. An examination of the item factor loadings confirmed the appropriateness of the six-factor solution suggested by Cattell's scree-test. ${ }^{11}$ The six-factor structure accounted for $34 \%$ of the response variance. A careful analysis of item content was used to identify the constructs represented by the empirically determined structure. The first three factors were clearly identified with problems experienced by the respondents. They were labeled "Control Problems," "Psychosocial Impact," and "Barriers to Compliance." The remaining factors were labeled "Benefits of Regimen," "Regimen Complexity," and "Risk of Complications." Table 2 contains samples of items from each of these factors. The factor associated with regimen complexity was correlated with control problems (.32) and with benefits of regimen (.22). No other significant correlation among factors was found.

A confirmatory factor analysis of the field test data was done
Table 2. Sample Items from Each Factor of DEP

\section{Control Problems}

Sometimes people, by accident or on purpose, change how much diabetes medication they take. Have you ever changed the amount or skipped your insulin/diabetes pills because:

... you forgot about your medicine?

you wanted to experiment?

.. you were upset about something?

... you ate too much food?

Psychosocial Impact

My diabetes and its treatment keep me from:

. having enough money.

doing my work and other responsibilities.

going out or traveling as much as I want.

being as active as I want.

... having good relationships with people.

having a schedule I like (eg, sleeping late).

\section{Barriers to Compliance}

When you have trouble with your diet, how often is it because:

. you are still hungry after eating?

you are eating away from home?

. you crave food you should not eat?

it is a special occasion (eg, birthday, holiday)?

\section{Benefits of Regimen}

How much does each of the following help you control your diabetes? . following your meal plan (eating the right foods at the right times). getting enough exercise.

testing urine as often as instructed.

\section{Regimen Complexity}

Are you supposed to follow a diet to control your diabetes or to lose weight? Have you been told to follow a schedule for your meals and snacks? Are you supposed to weigh or measure your food?

Have you been told to use exchange lists (food groups) to plan your meals? Do you (or the person who cooks your food) use the food groups (exchange lists) to plan your meals?

How often are you able to follow your meal plan closely (ie, you eat the right types and amounts of food at the right times)?

\section{Risks of Complications}

If I stopped taking care of my diabetes: I could get eye problems.

on the first 258 administrations obtained in the representative community sample. An oblique rotation ${ }^{12}$ of the factor matrix was used to approximate, insofar as possible, the structure obtained from the previous analysis of pilot data. Kaiser's $\mathrm{MSA}^{13}$ was used as a measure of sampling adequacy. A value of .92 was obtained. Results confirmed the earlier analysis but were not identical. The two matrices were compared by calculating congruence coefficients ${ }^{14}$ between item loadings for each sample. Excellent agreement was obtained across all factors. The congruence coefficients ranged from .86 to .74 (mean $=.82)$. Scale reliabilities were virtually identical between the two samples.

Reliability and Scoring Reliability estimates for the DEP subscales were based on internal consistency as expressed by Cronbach's coefficient alpha. Table 3 contains the estimated reliability of each factor, calculated using data representing both pilot and field study samples. As can be seen, the relia- 
bilities were high, ranging from .69 to .86 . Although stepwise item-total correlations were calculated to assess reliability with fewer items in some of the scales, elimination of items did not increase reliabilities and would alter the content (diet, medication, monitoring, and others) upon which the score was based. All DEP factor scores were standardized using the 428-case randomly selected sample as a norming group. Worksheets that included tables to convert raw scores into standardized scores were developed to allow hand-scoring of the instrument.

Validity The content validity of the instrument is based on the process of instrument development previously described. The list of characteristics related to the educational needs of patients with diabetes was rated by over 800 diabetes educators. Final item selection and arrangement into a cohesive conceptual structure were performed by a five-member review panel. Therefore, the process of instrument development ensured a high degree of content validity in the final instrument.

The construct validity of the instrument was demonstrated by the close agreement between the structure of the instrument (as described by factor analysis) and the planned structure, based on a variant of the Health Belief Model. Table 4 compares the relationship between the item content of the planned instrument structure (rows) and the empirically determined structure (columns). No general compliance factor was evident in the factor analysis, rather the planned compliance items loaded with the regimen, control problem, or benefits factors. The congruence coefficients for the six factors, which can be interpreted as correlations, ranged from .64 to 1.0; the mean value was .78 .

Some evidence for criterion validity is provided in Table 5 , which demonstrates statistically significant correlations between a measure of adherence (self-report) and five of the DEP factor scores. All of the DEP factors were correlated significantly with glycosylated hemoglobin and all but one were correlated with hospital admissions for diabetes within the previous two years. Social problems and barriers to adherence were each correlated with percentage of ideal body weight. It must be stressed that there are real differences in construct between these measures and the DEP factors, and it is believed that the magnitude of the correlations displayed is considerably reduced by other factors. These data are intended only to indicate the existence of a relationship.

Presentation of Individual Profiles An example of the DEP profile is reproduced as Figure 1. The top third of the form presents demographic information together with data on prescribed regimen, health status, and educational history needed to characterize the patient. The bottom third of the form provides space for the clinician to make notes regarding management activities to be changed or initiated.

The central portion of the form, of primary interest to readers of this article, presents an individual's scores on the six psychosocial scales as a profile. These scores have been standardized to simplify comparisons between the individual profiled and the continuum of others with diabetes. The 428-case randomly selected sample was used as a norming group. Since it is familiar to most health care professionals, the standardization convention used by the National Board of Medical Examiners (mean of 500, standard deviation of 100 ) was adopted
Table 3. Reliability Estimates for DEP Scales Based on Item Consistency as Measured by Cronbach's Coefficient Alpha

$\begin{array}{lc}\text { Factor } & \text { Alpha } \\ \text { Control problems } & .85 \\ \text { Psychosocial problems } & .84 \\ \text { Barriers to adherence } & .69 \\ \text { Benefits of regimen } & .77 \\ \text { Regimen complexity } & .85 \\ \text { Risk of complications } & .86\end{array}$

Table 4. Relationship Between Planned Structure and Empirically Determined Structure

\begin{tabular}{lcccccc}
\hline & Control & \multicolumn{2}{c}{ Social } & & & \\
Plan & Problems & Problems & Barriers & Benefits & Regimen Risk \\
History & .72 & - & - & - & .14 & - \\
Social & - & .81 & .32 & .07 & - & - \\
Barriers & .08 & .08 & .64 & .10 & - & - \\
Benefits & - & - & - & .77 & - & - \\
Behaviors & .08 & - & - & - & .73 & - \\
Risk & - & - & - & - & - & 1.00 \\
Compliance & .30 & - & - & .19 & .37 & - \\
\hline
\end{tabular}

Table 5. Correlations Between DEP Scores and Measures of Self-Care Behavior and Control

\begin{tabular}{lccccccc} 
& Control & \multicolumn{2}{c}{ Social } & & & & \\
Outcome & Problems & Problems & Barriers & Benefits & Regimen & Risk \\
Follow meal & & & & & & & \\
plan & -.07 & $-.11^{*}$ & $-.29 \dagger$ & $.25 \dagger$ & $.28 \dagger$ & $.11^{*}$ \\
$\begin{array}{l}\text { Glycosylated } \\
\text { hemoglobin }\end{array}$ & $.39 \dagger$ & $.21 \dagger$ & $.14 \dagger$ & $.16 \dagger$ & $.29 \dagger$ & $.10^{*}$ \\
Admissions & $.36 \dagger$ & $.24 \dagger$ & .03 & $.17 \dagger$ & $.29 \dagger$ & $.14 \dagger$ \\
$\%$ ideal weight & .00 & $.16 \dagger$ & $.22 *$ & .01 & .09 & .00 \\
& & & & & & & \\
$* p<.05$ & & & & & & \\
$+p<.01$ & & & & & & \\
\hline
\end{tabular}




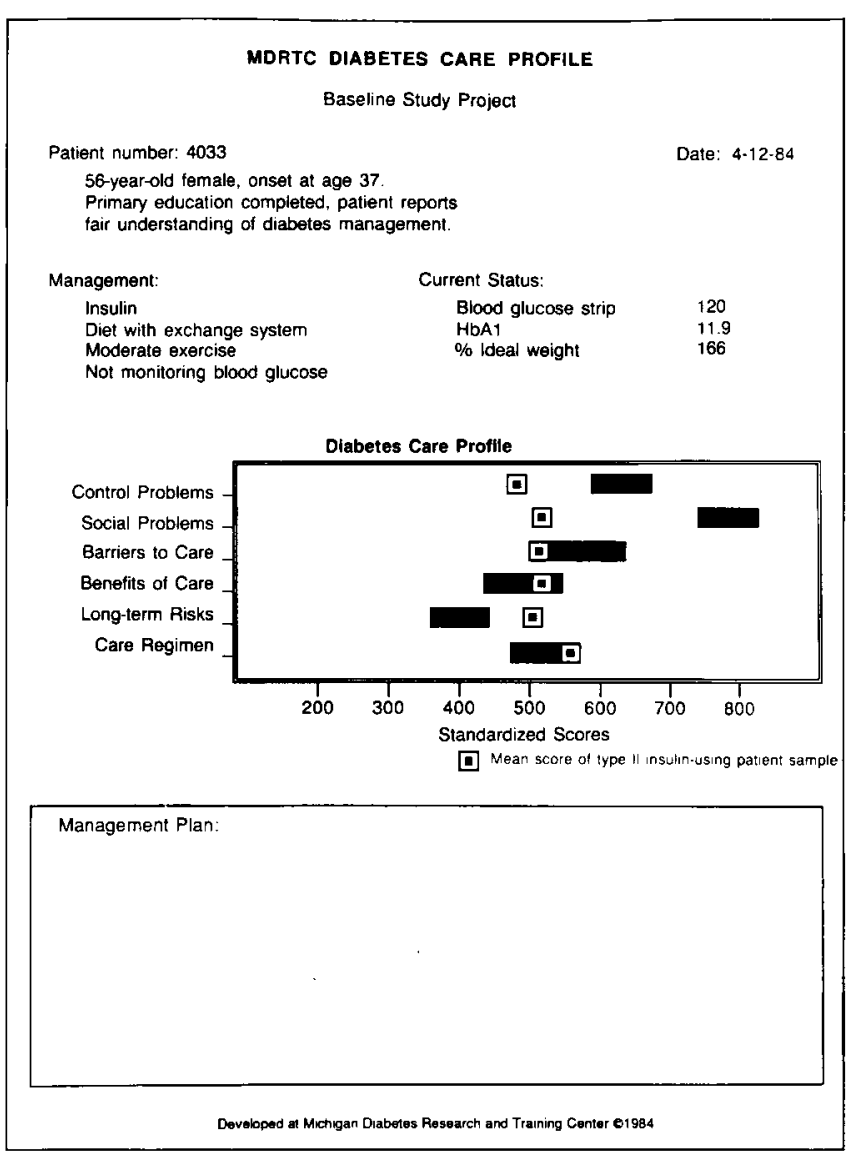

Fig 1. Example of DEP profile.

for the DEP. To clarify the significance of individual scores, these were plotted graphically as a bar extending one standard error of measurement in each direction from the obtained score. This bar represents the probably range of an error-free true score; thus, if any part of a profile bar was plotted over the 500 mark, the score could be interpreted as average. Bars extending to the right or left of this mark would be interpreted as above or below average, respectively. The extent to which an individual score is unusually high or low on a factor can be seen easily on the profile.

The small square plotted near the center of each scale provides more refined comparative information concerning the individual. Separate means for each factor have been calculated for three important groups of patients: (1) those with type I diabetes, (2) those with type II diabetes who use insulin, and (3) those with type II diabetes treated with oral agents or diet alone. As might be expected, there are significant differences in scores obtained from these groups. The group to which a person belong sis indivated immediately beneath the graphic portion of the form, and the means for this group are superimposed over the plot of individual scores. This feature of the

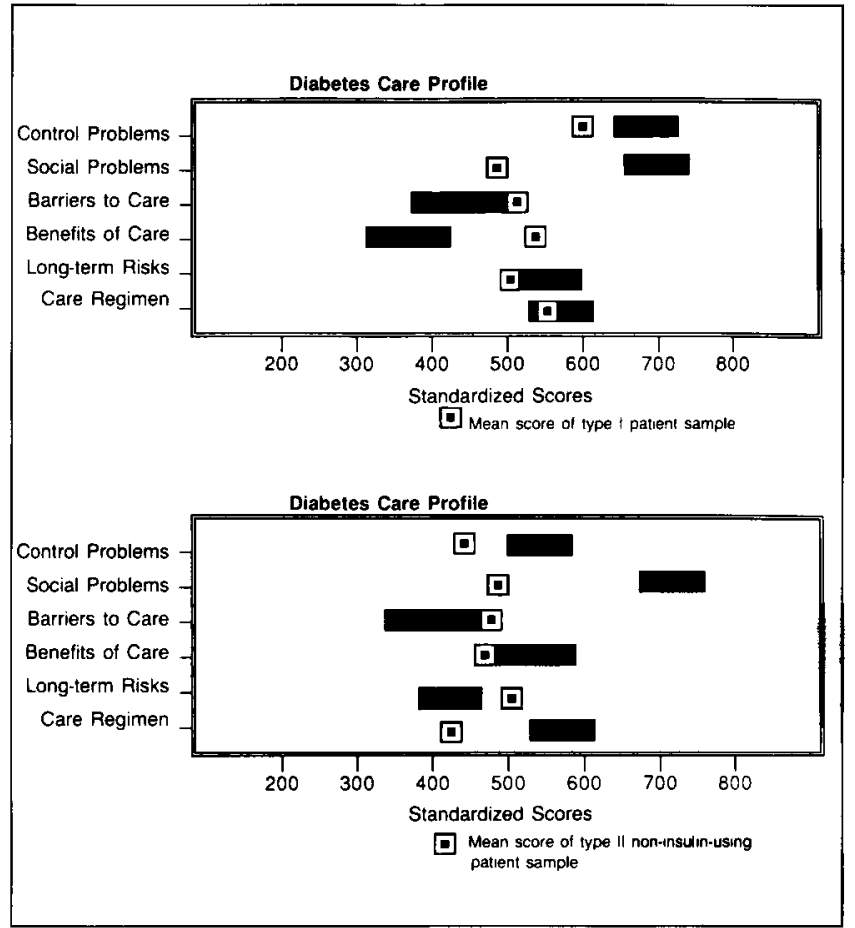

Fig 2. Example of two DEP profiles-different disease types and management regimens.

profile facilitates direct comparison between an individual's performance on the assessment and that of others with similar disease type and regimen. If the profile bar is clearly above or below this marker, as it is for five of the six factors shown in Figure 1, then careful consideration should be given to the direction of the departure and its implications for management and instruction.

In summary, for each factor score two comparisons can be made-one to a sample believed representative of all individuals with diabetes, and the other (and more relevant) one to a sample of clinically similar patients. Using this second scheme, an abnormal score is indicated by a separation between the plotted group mean and the score bar. This presentation immediately highlights abnormal scores and provides demographic, management, and current status information to consider in developing patient education and management plans.

\section{Discussion}

The use of factor analysis as a determinant of the final structure of the instrument ensured that the scales produced has 
optimum reliability. Given the close correspondence between the planned and the empirically determined structures, the included items are believed to measure the desired patient characteristics. It is also clear that the items rejected as a result of the analysis failed to achieve meaningful discrimination within the samples studied. Of particular interest was that items planned to assess compliance actually clustered at a more basic level; for example, control problems and regimen.

The decision to adopt the structure resulting from the analysis of the pilot sample rather than the random sample was based on the hypothesis that the pilot sample was more representative of those actively seeking medical assistance in managing their diabetes and, therefore, more likely to be candidates for the assessment. No differences in factor structure or reliability of the scales were found between the two samples.

The DEP provides the practicing health professional a convenient, easily administered instrument to assist in setting priorities for individualized counseling and instruction of patients. For the most part, the questionnaires can be selfadministered and are easily scored, either by hand or by using a customized computer program. Experience with the profile suggests that computerized scoring and profile generation are faster, more accurate, and more flexible in application. ${ }^{15}$

Even though most diabetes patient education programs rely heavily on group instruction, review of individual profiles can provide instructors with sound data for guidance in modifying curricula to meet the needs of class members. The profile also provides a useful focus for health care providers in assessing patient needs and attitudes regarding management regimens and health behaviors. Figure 2 contains the graphic portion of two profiles. Although both profiles highlight several problem areas, note that each patient is compared to the mean values representing his/her particular disease type and management regimen. The purpose of this profile is to elicit a specific plan to assist each individual in complying with his/her therapeutic regimen and in coping with the impact of a chronic disease.

The objectives of the instrument are primarily diagnostic. Since is does not provide specific guidance in how to resolve the problems identified by its use, problem resolution will continue to require the best professional insight and skill. Two limitations are associated with administration and scoring. First, data gathering is affected, to some extent, by the patients' reading ability and willingness to disclose information. Second, although hand-scoring of the profile is easily done, it can be laborious when large numbers of patients are involved and it lacks the more sophisticated presentation favored by the investigators. Computer-generated output, if available, is convenient and accurate, and allows for a variety of presentation formats.

The DEP was designed as an instructional needs assessment instrument for use by individual practitioners. An unexpected finding is that its major use to date has been as a research instrument in program evaluations and studies of regional diabetic populations. Although such applications of the instrument provide useful norms against which individual scores may be compared, no systematic collection of data summarizing instructional application of the DEP is currently available. Future efforts will center on increased application of the DEP and other assessment instruments to determine the educational and counseling needs of patients with diabetes and as a tool in evaluating the impact of these programs. These applications have been supported by development of a microcomputerbased program to score the instrument and generate graphic profiles and reports, thus making them immediately available for application.

Another result of ongoing instrument development activities is the addition of new items and expanded response options that reflect changes in diabetes management practices initiated since the instrument was developed. The revised instrument (Diabetes Care Profile) is similar to the one reported here, differing mainly in that it incorporates factors assessing social support and adherence to an individual's prescribed regimen. Pilot testing of the revised instrument is just being completed. A final version of the Diabetes Care Profile and a scoring program for IBM PC-compatible microcomputers became available for general use early in 1986 .

\section{References}

1. Surwit RS, Scovern AW, Feinglos MN: The role of behavior in diabetes care. Diabetes Care 5:337-342, 1982.

2. Surwit RS, Feinglos MN, Scovem AW: Diabetes and behavior: A paradigm for health psychology. Am Psychol 255-262, 1983.

3. Dunbar JM, Stunkard AJ: Adherence to diet and dnug regimen, in Levy R, Rifkind B, Dennis B, et al (eds): Nutrition, Lipids, and Coronary Heart Disease, New York, Raven Press, 1979.

4. Dudley JD: The diabetes educator's role in teaching the diabetic patient. Diabetes Care 3:127-131, 1980.

5. Skyler JS: Psychological issues in diabetes. Diabetes Care 4:656-7, 1981.

6. Glasgow RE, McCaul KD: Psychological issues in diabetes: A different approach. Diabetes Care 5:645-646, 1982.

7. Maiman LA, Becker MH: The Health Belief Model: Origins and correlates in psychological theory, in Becker MH (ed): The Health Belief Model and Personal Health Behavior. Health Education Monographs 2:4 pp 336-353, 1974.

8. Davis W, Hull AL, Boutaugh ML: Factors affecting the educational diagnosis of diabetic patients. Diabetes Care 4:275-278, 1981.

9. Hiss RG, Boutaugh ML, Helms BG, et al: Structure, process, and outcome-A basic approach to assessment of diabetes care, in Mazze RS (ed): Professional Education in Diabetes, pp 151-164, 1980.

10. Cronbach LJ: Coefficient alpha and the internal structure of tests. Psychometrica 16:297-337, 1951

11. Cattell RB: Factor analysis: An introduction to essentials. Biometrics 21:190-215, 405-435, 1965.

12. Mulaic SA: The Foundations of Factor Analysis. New York, McGrawHill, 1972, p 299.

13. Kaiser HF: Little jiffy, Mark IV. Educ and Psychol Meas 34:111-117, 1974.

14. Burt C: The factorial study of temperamental traits. Brit J Psych, Statistical Section 1:178-203, 1948.

15. Hess GE, Davis WK: Microcomputer-based scoring and display of behavioral profiles. Diabetes 33:385, 1984. 